\title{
GENERALIZED DEMAZURE MODULES AND PRIME REPRESENTATIONS IN TYPE $D_{n}$
}

\author{
VYJAYANTHI CHARI, JUSTIN DAVIS AND RYAN MORUZZI JR.
}

To Kolya Reshetikhin, on his 60th birthday.

\begin{abstract}
The goal of this paper is to understand the graded limit of a family of irreducible prime representations of the quantum affine algebra associated to a simply-laced simple Lie algebra $\mathfrak{g}$. This family was introduced in [19, 20, in the context of monoidal categorification of cluster algebras. The graded limit of a member of this family is an indecomposable graded module for the current algebra $\mathfrak{g}[t]$; or equivalently a module for the maximal standard parabolic subalgebra in the affine Lie algebra $\widehat{\mathfrak{g}}$. In the case when $\mathfrak{g}$ is of type $A_{n}$ the problem was studied in [4, where it was shown that the graded limit is isomorphic to a level two Demazure module. In this paper we study the case when $\mathfrak{g}$ is of type $D_{n}$. We show that in certain cases the limit is a generalized Demazure module, i.e., it is a submodule of a tensor product of level one Demazure modules. We give a presentation of these modules and compute their graded character (and hence also the character of the prime representations) in terms of Demazure modules of level two.
\end{abstract}

\section{INTRODUCTION}

Kirillov-Reshetikhin modules (or KR-modules) are a family of finite-dimensional modules for the quantum affine algebra associated to a simple Lie algebra. These modules were introduced and originally studied by Kirillov and Reshetikhin in [22] in connection with the closely related Yangians. The interest in these representations arose from their study of solvable lattice models which allowed them to formulate an important conjecture on the character of the tensor products of these modules. Subsequently, with the introduction of $q$-characters in [14, which was further developed in [12, 13, it became more natural and more tractable to study the Kirillov-Reshetikhin modules for the quantum affine algebra associated to a simple Lie algebra. In [29, 30] the Kirillov-Reshetikhin conjecture was solved by geometric methods when $\mathfrak{g}$ is simply-laced. The non-simply laced case was done in 18 using a $q$-character approach. A combinatorial version of this conjecture was proved in [11]. A crystal basis approach to these modules was first developed in [16, 17] and there is a vast literature on crystal bases for these modules (see for instance [24, 37]). We emphasize here that our references to the topics discussed in the introduction are nowhere near comprehensive, that would require a separate volume!

One approach to understanding the character of KR-modules and more generally the character of finite-dimensional representations of quantum affine algebras is to study the classical $(q \rightarrow 1)$ limit of these modules. The systematic study of this approach was begun in [9], where

VC was partially supported by DMS- 1719357, the Simons Fellows Program and by the Infosys Visiting Chair position at the Indian Institute of Science. 
a necessary and sufficient condition for the existence of the limit was proved. All the interesting representations studied in the literature admit a classical limit. The limit, when it exists, is a finite-dimensional module for the corresponding affine Lie algebra and so also a module for current algebra $\mathfrak{g}[t]$ which is the Lie algebra of polynomial maps from $\mathbb{C} \rightarrow \mathfrak{g}$. The notion of the graded limit of a representation of a quantum affine algebra was developed in [5, 8]. We refer the reader to [21] for a discussion of the role of graded limits in the KR-conjecture. An interesting problem is to give a presentation of the graded limit and to compute its graded character; this is particularly so, because a presentation and the character of an irreducible finite-dimensional module for the quantum affine algebra is not known in general. This problem was studied for a class of representations called minimal affinizations in [27, 33, 34] when $\mathfrak{g}$ is of classical type and in [25, 28] for some exceptional types.

D. Hernandez and B. Leclerc introduced in [19, 20] the notion of a monoidal categorification of cluster algebras. They defined certain tensor subcategories of representations of the quantum affine algebra and showed that their Grothendieck ring was a cluster algebra of Dynkin type. The cluster variables are particular 'prime' irreducible modules (which we shall refer to as HL-modules) of the quantum affine algebra and a cluster monomial is an irreducible tensor product of HL-modules. (Recall that a representation is said to be prime if it is not isomorphic to a tensor product of non-trivial representations). The HL-modules include some of the well-known ones, such as certain KR-modules, but also include many new examples of nonisomorphic prime irreducible representations which are not well-understood.

We are interested in the graded limit of HL-modules. In the case of $A_{n}$ this problem was studied in [3, 4] and we discuss this briefly. The results of those papers imply that the graded limit of an HL-module is isomorphic to a Demazure module occurring in a level two representation of the affine Lie algebra. The graded character of Demazure modules is known to be given by the Demazure character formula. In recent work [1, the graded characters of HLmodules in type $A_{n}$ are given explicitly in terms of Macdonald polynomials. The appearance of level one Demazure modules as graded limits goes back to the work of [7, 15, 32] and we say no more about it here.

In this paper we are interested in the limits of HL-modules in type $D_{n}$. We restrict our attention to the modules which correspond to the roots of $D_{n}$ in which the simple roots occur with multiplicity at most one. Even with this restriction, the situation is substantially more complicated than $A_{n}$. We prove that the graded limit of an HL-module is a generalized Demazure module, i.e. it occurs as a particular submodule of the tensor product of level one Demazure modules. The study of generalized Demazure modules is not very well-developed and in particular, no presentation or character formula is known in general for these modules. Thus, in the first two sections we work entirely in the context of the current algebra $\mathfrak{g}[t]$ where $\mathfrak{g}$ is of type $D_{n}$ and give a presentation and a graded character formula for a family of generalized Demazure modules. In the final section of the paper we explain briefly the connection with the work of Hernandez and Leclerc. These connections are similar to the $A_{n}$ case which is explained in great detail in [3, 4. There are difficulties which arise when we take the HL-modules associated with the remaining cluster variables; namely those which involve a simple root with multiplicity two. However, it looks likely that these will have a flag where the successive quotients are level two Demazure modules and we hope to study this elsewhere. 


\section{Generalized Demazure Modules}

In this section we set up the basic notation and define the Demazure and generalized Demazure modules associated with an integrable highest weight representation of the untwisted affine Lie algebra $D_{n}^{(1)}$. In the latter part of the section we state and prove the main result assuming two key steps in the proof.

1.1. Throughout this paper $\mathfrak{g}$ will denote a simple Lie algebra of type $D_{n}$ and $\mathfrak{h}$ a fixed Cartan subalgebra. Let $\kappa: \mathfrak{g} \times \mathfrak{g} \rightarrow \mathbb{C}$ be the Killing form on $\mathfrak{g}$ and $():, \mathfrak{h}^{*} \times \mathfrak{h}^{*} \rightarrow \mathbb{C}$ be the induced symmetric bilinear form on $\mathfrak{h}^{*}$. Let $R$ and $W$ denote the set of roots and the Weyl group respectively of the pair $(\mathfrak{g}, \mathfrak{h})$. Fix a set of simple roots $\left\{\alpha_{i}: 1 \leq i \leq n\right\}$ where $\alpha_{n-1}, \alpha_{n}$ are the spin nodes and $\alpha_{n-2}$ is the trivalent node and fix a set of fundamental weights $\left\{\omega_{i}: 1 \leq i \leq n\right\}$ satisfying $\left(\omega_{i}, \alpha_{j}\right)=\delta_{i, j}$. It will be convenient to set $\omega_{0}=0=\omega_{n+1}$. As usual we let $Q$ and $P$ be the integer span of the simple roots and fundamental weights respectively; the sets $Q^{+}$and $P^{+}$are defined in the obvious way and we set $R^{+}=R \cap Q^{+}$.

Set,

$$
\begin{gathered}
\alpha_{i, j}=\alpha_{i}+\cdots+\alpha_{j}, \quad \alpha_{i, n}=\alpha_{i}+\cdots+\alpha_{n-2}+\alpha_{n}, \quad 1 \leq i \leq j \leq n-1, \\
\beta_{i, j}=\alpha_{i}+\cdots+\alpha_{j-1}+2\left(\alpha_{j}+\cdots+\alpha_{n-2}\right)+\alpha_{n-1}+\alpha_{n}, \quad 1 \leq i<j \leq n-1, \\
h_{i, j}=h_{i}+\cdots+h_{j}, \quad 1 \leq i \leq j \leq n-1, \\
h_{i, n}=h_{i}+h_{i+1}+\cdots+h_{n-2}+h_{n}, \quad 1 \leq i \leq n-2 .
\end{gathered}
$$

We shall adopt the convention that $\alpha_{i, j}=h_{i, j}=0$ if $i>j$ or if $(i, j)=(n-1, n)$. Note that

$$
R^{+}=\left\{\alpha_{i, j}: 1 \leq i \leq j \leq n\right\} \sqcup\left\{\beta_{i, j}: 1 \leq i<j \leq n-1\right\} .
$$

Define a partial order on $P$ by $\lambda \leq \mu$ iff $\mu-\lambda \in Q^{+}$. Finally, let $\left\{x_{\alpha}^{ \pm}, h_{i}: \alpha \in R^{+}, i \in I\right\}$ be a Chevalley basis of $\mathfrak{g}$ and set $x_{\alpha_{i}}^{ \pm}=x_{i}^{ \pm}$. Let $\mathfrak{g}=\mathfrak{n}^{-} \oplus \mathfrak{h} \oplus \mathfrak{n}^{+}$be the corresponding triangular decomposition.

1.2. Let $\widehat{\mathfrak{g}}$ be the untwisted affine Lie algebra associated to $\mathfrak{g}$ and recall that it has the following explicit realization. Given an indeterminate $t$, let $\mathbb{C}\left[t, t^{-1}\right]$ be the algebra of Laurent polynomials and set

$$
\widehat{\mathfrak{g}}=\mathfrak{g} \otimes \mathbb{C}\left[t, t^{-1}\right] \oplus \mathbb{C} c \oplus \mathbb{C} d .
$$

Define the commutator on $\widehat{\mathfrak{g}}$ by requiring $c$ to be central, and

$$
\left[x \otimes t^{r}, y \otimes t^{s}\right]=[x, y] \otimes t^{r+s}+r \delta_{r+s, 0} \kappa(x, y) c, \quad\left[d, x \otimes t^{r}\right]=r\left(x \otimes t^{r}\right), \quad x, y \in \mathfrak{g}, \quad r, s \in \mathbb{Z} .
$$

Then

$$
\widehat{\mathfrak{h}}=\mathfrak{h} \oplus \mathbb{C} c \oplus \mathbb{C} d
$$

is a Cartan subalgebra of $\widehat{\mathfrak{g}}$ and we extend an element $\lambda \in \mathfrak{h}^{*}$ to an element of $\widehat{\mathfrak{h}}^{*}$ by setting $\lambda(c)=0=\lambda(d)$. Define elements $\Lambda_{0}, \delta, \alpha_{0} \in \widehat{\mathfrak{h}}^{*}$ by

$$
\Lambda_{0}(\mathfrak{h} \oplus \mathbb{C} d)=0, \quad \Lambda_{0}(c)=1, \quad \delta(\mathfrak{h} \oplus \mathbb{C} c)=0, \quad \delta(d)=1, \quad \alpha_{0}=-\beta_{1,2}+\delta .
$$

The set $\left\{\alpha_{i}: 0 \leq i \leq n\right\}$ is a set of simple roots for the pair $(\widehat{\mathfrak{g}}, \widehat{\mathfrak{h}})$ and the corresponding set of affine fundamental weights is given by $\left\{\Lambda_{i}: 0 \leq i \leq n\right\}$ where

$$
\Lambda_{i}=\omega_{i}+a_{i}^{\vee} \Lambda_{0}, \quad a_{i}^{\vee}=2, \quad 2 \leq i \leq n-2, \quad a_{i}^{\vee}=1, \quad i=1, n-1, n .
$$


Let $\widehat{W}$ be the affine Weyl group and note that it contains an isomorphic copy of $W$. Define the affine root lattice $\widehat{Q}$ and affine weight lattice $\widehat{P}$ and the subsets $\widehat{Q}^{+}, \widehat{P}^{+}$and the set of affine positive roots in the natural way. Let

$$
\widehat{\mathfrak{b}}=\widehat{\mathfrak{h}} \oplus\left(\mathfrak{n}^{+} \otimes 1\right) \oplus(\mathfrak{g} \otimes t \mathbb{C}[t]), \quad \widehat{\mathfrak{p}}=\left(\mathfrak{n}^{-} \otimes 1\right) \oplus \widehat{\mathfrak{b}}
$$

be the Borel subalgebra and the standard maximal parabolic subalgebra of $\widehat{\mathfrak{g}}$ with respect to $\widehat{R}^{+}$. Let $\widehat{\mathfrak{n}}^{+}$be the commutator subalgebra of $\widehat{\mathfrak{b}}$ and note that the commutator subalgebra of $\widehat{\mathfrak{p}}$ is just the current algebra $\mathfrak{g}[t]=\mathfrak{g} \otimes \mathbb{C}[t]$ associated to $\mathfrak{g}$. The action of $d$ induces a canonical $\mathbb{Z}_{+^{-}}$grading on $\mathfrak{g}[t]$ and on its universal enveloping algebra with the grade of an element $x \otimes t^{r} \in \mathfrak{g}[t]$ being $r$.

1.3. Given $\Lambda \in \widehat{P}^{+}$let $\widehat{V}(\Lambda)$ be the integrable irreducible $\widehat{\mathfrak{g}}$-module generated by an element $\widehat{v}_{\Lambda}$ with relations

$$
\begin{gathered}
\widehat{\mathfrak{n}}^{+} \widehat{v}_{\Lambda}=0, \quad h \widehat{v}_{\Lambda}=\Lambda(h) \widehat{v}_{\Lambda}, \quad h \in \widehat{\mathfrak{h}}, \\
\left(x_{i}^{-} \otimes 1\right)^{\Lambda\left(h_{i}\right)+1} \widehat{v}_{\Lambda}=0, \quad 1 \leq i \leq n, \quad\left(x_{\beta_{1,2}}^{+} \otimes t^{-1}\right)^{\Lambda\left(h_{0}\right)+1} \widehat{v}_{\Lambda}=0 .
\end{gathered}
$$

We have

$$
\widehat{V}(\Lambda)=\bigoplus_{\Lambda^{\prime} \in \widehat{P}} \widehat{V}(\Lambda)_{\Lambda^{\prime}}, \quad \widehat{V}(\Lambda)_{\Lambda^{\prime}}=\left\{v \in \widehat{V}(\Lambda): h v=\Lambda^{\prime}(h) v, \quad h \in \widehat{\mathfrak{h}}\right\}
$$

It is known that

$$
\operatorname{dim} \widehat{V}(\Lambda)_{w \Lambda}=1, \text { for all } w \in \widehat{W},
$$

and we let $\widehat{V}_{w}(\Lambda)$ be the $\widehat{\mathfrak{b}}$-module generated by $\widehat{V}(\Lambda)_{w \Lambda}$. The modules $\widehat{V}_{w}(\Lambda)$ are finitedimensional and are called the Demazure modules associated to $\Lambda$. Moreover $\widehat{v}_{\Lambda} \in \widehat{V}_{w}(\Lambda)$ for all $w \in \widehat{W}$. The following is now essentially immediate.

Lemma. Suppose that $w, w^{\prime} \in \widehat{W}$ and $\Lambda \in \widehat{P}^{+}$. Then

$$
\operatorname{dim} \operatorname{Hom}_{\widehat{\mathfrak{b}}}\left(\widehat{V}_{w}(\Lambda), \widehat{V}_{w^{\prime}}(\Lambda)\right) \leq 1,
$$

and any non-zero element of this space is injective.

1.4. Given $\Lambda, \Lambda^{\prime} \in \widehat{P}^{+}$and $w, w^{\prime} \in \widehat{W}$ we set

$$
\widehat{V}_{w, w^{\prime}}\left(\Lambda, \Lambda^{\prime}\right)=\mathbb{U}(\widehat{\mathfrak{b}})\left(\widehat{V}(\Lambda)_{w \Lambda} \otimes \widehat{V}\left(\Lambda^{\prime}\right)_{w^{\prime} \Lambda^{\prime}}\right) .
$$

These (along with an obvious extension to an arbitrary number of dominant integral weights and Weyl group elements) are called the generalized Demazure modules. Further, since

$$
\operatorname{dim} \operatorname{Hom}_{\widehat{\mathfrak{g}}}\left(\widehat{V}(\Lambda) \otimes \widehat{V}\left(\Lambda^{\prime}\right), \widehat{V}\left(\Lambda+\Lambda^{\prime}\right)\right)=1
$$

it is easy to see that

$$
\widehat{V}(\Lambda)_{w \Lambda} \otimes \widehat{V}\left(\Lambda^{\prime}\right)_{w \Lambda^{\prime}} \cong \widehat{V}\left(\Lambda+\Lambda^{\prime}\right)_{w\left(\Lambda+\Lambda^{\prime}\right)} \text { and so } \widehat{V}_{w, w}\left(\Lambda, \Lambda^{\prime}\right) \cong \widehat{V}_{w}\left(\Lambda+\Lambda^{\prime}\right) .
$$

In this paper we shall be interested in certain special families of Demazure and generalized Demazure modules; namely those associated to pairs $(w, \Lambda)$ such that the restriction of $w \Lambda$ to $\mathfrak{h}$ is in $\left(-P^{+}\right)$. This restriction guarantees that $\widehat{V}_{w}(\Lambda)$ is stable under $\widehat{\mathfrak{p}}^{+}$. Equivalently, we can (and do) regard these modules as $\mathbb{Z}$-graded modules for $\mathfrak{g}[t]$ and call them the stable Demazure modules. We recall a result proved independently in [23, 26]. 
Theorem. Let $\Lambda, \Lambda^{\prime} \in \widehat{P}^{+}$and $w, w^{\prime} \in \widehat{W}$. If $\sigma \in \widehat{W}$ is such that $\sigma\left(w \Lambda+w^{\prime} \Lambda^{\prime}\right) \in \widehat{P}^{+}$, then there exists a projection of $\widehat{\mathfrak{g}}$-modules

$$
\widehat{V}(\Lambda) \otimes \widehat{V}\left(\Lambda^{\prime}\right) \longrightarrow \widehat{V}\left(\sigma\left(w \Lambda+w^{\prime} \Lambda^{\prime}\right)\right) \rightarrow 0,
$$

which induces an isomorphism of the one-dimensional spaces

$$
\widehat{V}(\Lambda)_{w \Lambda} \otimes \widehat{V}\left(\Lambda^{\prime}\right)_{w^{\prime} \Lambda^{\prime}} \cong \widehat{V}\left(\sigma\left(w \Lambda+w^{\prime} \Lambda^{\prime}\right)\right)_{w \Lambda+w^{\prime} \Lambda^{\prime}}
$$

The following corollary is immediate from the preceding theorem and is needed later in this section.

Corollary. Retain the notation of the theorem. Assume that $\widehat{V}_{w}(\Lambda)$ and $\widehat{V}_{w^{\prime}}\left(\Lambda^{\prime}\right)$ are stable Demazure modules. Then $\widehat{V}_{\sigma^{-1}}\left(\sigma\left(w \Lambda+w^{\prime} \Lambda^{\prime}\right)\right)$ is also a stable Demazure module and there exists a surjective map of $\widehat{\mathfrak{p}}$-modules

$$
\widehat{V}_{w, w^{\prime}}\left(\Lambda, \Lambda^{\prime}\right) \rightarrow \widehat{V}_{\sigma^{-1}}\left(\sigma\left(w \Lambda+w^{\prime} \Lambda^{\prime}\right)\right) \rightarrow 0
$$

1.5. Let $w_{\circ}$ be the longest element in $W$. Suppose that $(w, \Lambda)$ and $\left(w^{\prime}, \Lambda^{\prime}\right)$ are such that $\Lambda(c)=\Lambda^{\prime}(c)=\ell$ and $\lambda=\left.w_{\circ} w \Lambda\right|_{\mathfrak{h}}=\left.w_{\circ} w^{\prime} \Lambda^{\prime}\right|_{\mathfrak{h}} \in P^{+}$. Then it is known [15, 32, that $V_{w}(\Lambda)$ is isomorphic to $V_{w^{\prime}}\left(\Lambda^{\prime}\right)$ as $\mathfrak{g}[t]$-modules up to an overall shift of the action of $d$. In particular we can regard the stable Demazure modules as being indexed by a pair $(\ell, \lambda) \in \mathbb{N} \times P^{+}$.

In what follows we prefer to regard the stable (generalized) Demazure modules as $\mathbb{Z}$-graded modules for the current algebra of $\mathfrak{g}[t]$, where the grading is given by the action of $d$. The maps between graded modules will be of degree zero. Given $s \in \mathbb{Z}$ and a graded $\mathfrak{g}[t]$-module we denote by $\tau_{s}^{*} V$ the graded $\mathfrak{g}[t]$-module obtained by shifting the grades by $s$.

We recall a presentation of the stable Demazure modules given in [10]. Let $D(\ell, \lambda)$ be the $\mathfrak{g}[t]$-module generated by an element $w_{\lambda}$ with the following defining relations: for $1 \leq i \leq n$, $\alpha \in R^{+}, h \in \mathfrak{h}$, we have

$$
\begin{gathered}
\mathfrak{n}^{+}[t] w_{\lambda}=0, \quad\left(h \otimes t^{r}\right) w_{\lambda}=\delta_{r, 0} \lambda(h) w_{\lambda}, \quad\left(x_{i}^{-} \otimes 1\right)^{\lambda\left(h_{i}\right)+1} w_{\lambda}=0, \\
\left(x_{\alpha}^{-} \otimes t^{s_{\alpha}}\right) w_{\lambda}=0, \quad\left(x_{\alpha}^{-} \otimes t^{s_{\alpha}-1}\right)^{m_{\alpha}+1} w_{\lambda}=0,
\end{gathered}
$$

where we write $\lambda\left(h_{\alpha}\right)=\ell\left(s_{\alpha}-1\right)+m_{\alpha}$ with $s_{\alpha} \in \mathbb{N}$ and $0<m_{\alpha} \leq \ell$. The $\mathbb{Z}$-grading on $D(\ell, \lambda)$ is given by requiring $w_{\lambda}$ to have grade zero.

In the case $\ell=1,2$ the relations can be further streamlined. The following was proved when $\ell=1$ in [9] and when $\ell=2$ in [4].

Lemma. If $\ell=1$ the relations in (1.2) are a consequence of the relations in (1.1). If $\ell=2$ then the second relation in (1.2) is a consequence of the others.

1.6. In the language of $\mathfrak{g}[t]$-modules developed above, a generalized Demazure module corresponds to considering a tensor product $\tau_{s}^{*} D(\ell, \lambda) \otimes \tau_{s^{\prime}}^{*} D\left(\ell^{\prime}, \lambda^{\prime}\right)$ and taking the $\mathfrak{g}[t]$-module through $w_{\lambda} \otimes w_{\lambda^{\prime}}$. Presentations of generalized Demazure modules have not been much studied although there is some work on the subject. They play an important role in the study of classical limits of representations of quantum affine algebras and first arose in this context in 
[33, 34] (see 36] for other examples). In this paper we shall give a presentation of a particular family of generalized Demazure modules, namely those of the form

$$
D(\lambda, \mu):=\mathbb{U}(\mathfrak{g}[t])\left(w_{\lambda} \otimes w_{\mu}\right) \subset D(1, \lambda) \otimes D(1, \mu),
$$

with some restrictions on the pair $(\lambda, \mu) \in P^{+} \times P^{+}$. Our motivations for considering this problem was discussed extensively in the introduction and further details can also be found in Section 3. We note the following reformulation of Corollary 1.4.

Lemma. There exists a (unique up to scalars) map $\eta_{\lambda, \mu}: D(\lambda, \mu) \rightarrow D(2, \lambda+\mu) \rightarrow 0$, of $\mathfrak{g}[t]$-modules extending the assignment $w_{\lambda} \otimes w_{\mu} \rightarrow w_{\lambda+\mu}$.

1.7. Given $\lambda, \mu \in P^{+}$, let $V(\lambda, \mu)$ be the $\mathfrak{g}[t]$-module generated by an element $w_{\lambda, \mu}$ satisfying the following defining relations:

$$
\begin{gathered}
\mathfrak{n}^{+}[t] w_{\lambda, \mu}=0, \quad\left(h \otimes t^{r}\right) w_{\lambda, \mu}=\delta_{r, 0}(\lambda+\mu)(h) w_{\lambda, \mu}, \quad\left(x_{i}^{-} \otimes 1\right)^{(\lambda+\mu)\left(h_{i}\right)+1} w_{\lambda, \mu}=0, \\
\left(x_{\alpha}^{-} \otimes t^{\max \left\{\lambda\left(h_{\alpha}\right), \mu\left(h_{\alpha}\right)\right\}}\right) w_{\lambda, \mu}=0,
\end{gathered}
$$

where $1 \leq i \leq n, h \in \mathfrak{h}$ and $\alpha \in R^{+}$. Define a grading on $V(\lambda, \mu)$ by requiring the grade of $w_{\lambda, \mu}$ to be zero. Clearly $V(\lambda, \mu)$ is a graded quotient of $D(1, \lambda+\mu)$ and $V(\lambda, \mu) \cong V(\mu, \lambda)$. Moreover Lemma 1.5 shows that if $\mu=0$ then $V(\lambda, 0) \cong D(1, \lambda)$. The following is immediate from equations (1.1)-(1.4) and Lemma 1.6.

Lemma. The assignments $w_{\lambda, \mu} \rightarrow w_{\lambda+\mu}$ and $w_{\lambda, \mu} \rightarrow w_{\lambda} \otimes w_{\mu}$ define surjective maps of graded $\mathfrak{g}[t]$-modules

$$
\psi_{\lambda, \mu}: V(\lambda, \mu) \rightarrow D(2, \lambda+\mu), \quad \varphi_{\lambda, \mu}: V(\lambda, \mu) \rightarrow D(\lambda, \mu) \rightarrow 0
$$

and $\psi_{\lambda, \mu}=\eta_{\lambda, \mu} \circ \varphi_{\lambda, \mu}$.

\subsection{Let}

$$
P^{+}(1)=\left\{\lambda \in P^{+}: \lambda\left(h_{i}\right) \leq 1, \quad 1 \leq i \leq n\right\} .
$$

It is obvious that any $\lambda \in P^{+}(1)$ can be written uniquely (up to order) as a sum $\lambda=\lambda_{1}+\lambda_{2}$ where $\lambda_{s} \in P^{+}(1), s=1,2$ and satisfy the following: for $1 \leq i \leq j \leq n$ and $(i, j) \neq(n-1, n)$

$$
\lambda_{r}\left(h_{i}\right)=1=\lambda_{r}\left(h_{j}\right) \Longrightarrow \lambda_{p}\left(h_{s}\right)=1 \text { for some } i<s<j, \quad\{r, p\}=\{1,2\},
$$

and

$$
\lambda\left(h_{n-1}+h_{n}\right)>0 \Longrightarrow \lambda_{p}\left(h_{n-1}+h_{n}\right)=0 \text { for some } p \in\{1,2\} .
$$

It will be convenient to call $\left(\lambda_{1}, \lambda_{2}\right) \in P^{+} \times P^{+}$an interlacing pair if $\lambda_{1}+\lambda_{2} \in P^{+}(1)$ and the preceding two conditions hold.

Examples. The pairs $\left(\omega_{i}, 0\right)$ for $0 \leq i \leq n,\left(\omega_{n-1}+\omega_{n}, 0\right)$ and the elements of the set $\left\{\left(\omega_{i}, \omega_{j}\right): 0 \leq i \neq j \leq n \quad(i, j) \neq(n-1, n)\right\}$ are interlacing. The pair $\left(\omega_{2}+\omega_{5}, \omega_{3}+\omega_{6}+\omega_{7}\right)$ is interlacing in $D_{7}$ but not in $D_{8}$. 
1.9. The following is the main result of this note.

Theorem. Let $\left(\lambda_{1}, \lambda_{2}\right) \in P^{+} \times P^{+}$be an interlacing pair. For all $\nu \in P^{+}$the map

$$
\varphi_{\lambda_{1}+\nu, \lambda_{2}+\nu}: V\left(\lambda_{1}+\nu, \lambda_{2}+\nu\right) \rightarrow D\left(\lambda_{1}+\nu, \lambda_{2}+\nu\right)
$$

is an isomorphishm. Moreover $D\left(\lambda_{1}+\nu, \lambda_{2}+\nu\right)$ admits a flag and the quotients are isomorphic to $\tau_{s}^{*} D(2, \mu)$ for some $s \in \mathbb{Z}_{+}, \mu \in P^{+}$.

Remark. One can give a precise formula for the graded character of $D\left(\lambda_{1}+\nu, \lambda_{2}+\nu\right)$ and this can be found in Section 1.8 .

Remark. An analogous result was proved for $A_{n}$ in [4] when $\nu=0$. In fact in that case it was proved that

$$
V\left(\lambda_{1}, \lambda_{2}\right) \cong D\left(\lambda_{1}, \lambda_{2}\right) \cong D\left(2, \lambda_{1}+\lambda_{2}\right) .
$$

Our methods are different and work for $A_{n}$ as well and prove the more general case when $\nu \neq 0$.

Remark. It is plausible that the map $\varphi_{\lambda, \mu}: V(\lambda, \mu) \rightarrow D(\lambda, \mu)$ is always an isomorphism. This is known to be true for $\mathfrak{s l}_{2}$ and examples exist in $\mathfrak{s l}_{n}$ (see for instance [2, 35]).

In the rest of the section we shall assume that $\left(\lambda_{1}, \lambda_{2}\right)$ is an interlacing pair and $\lambda=\lambda_{1}+\lambda_{2}$. Moreover the property of interlacing pairs means that we can and will assume without loss of generality that if $\lambda\left(h_{n-1}+h_{n}\right)>0$ then $\lambda_{1}\left(h_{n-1}\right)>0$. This means that if $p$ is maximal such that $\lambda\left(h_{p+1}\right)=1$ then $p \leq n-2$ and $\lambda_{1}\left(h_{p+1}\right)=1$ and $\lambda_{2}\left(h_{p+1}\right)=0$.

1.10. The theorem is proved in several steps. The first reduction is the following proposition which gives a condition for the generalized Demazure module to be isomorphic to a Demazure module.

Proposition. If $\lambda\left(h_{n-1}+h_{n}\right)=1$ or if $\lambda=\omega_{i-1}+\omega_{i}+\delta_{i+1, n} \omega_{n}$ for $1 \leq i \leq n-1$ then for all $\nu \in P^{+}$we have

$$
V\left(\lambda_{1}+\nu, \lambda_{2}+\nu\right) \cong D(2,2 \nu+\lambda) \cong D\left(\lambda_{1}+\nu, \lambda_{2}+\nu\right) .
$$

1.11. Suppose that $\lambda\left(h_{n-1}+h_{n}\right) \in\{0,2\}$ and $\lambda \neq \omega_{i-1}+\omega_{i}+\delta_{i+1, n} \omega_{n}$ and let $p \leq n-2$ be maximal such that $\lambda_{1}\left(h_{p+1}\right)=1=\lambda\left(h_{p+1}\right)$. Let $1 \leq p^{\prime} \leq p \leq n-2$ be maximal so that $\left(\lambda_{1}-\lambda_{2}\right)\left(h_{p^{\prime}, p}\right)=0$. Observe that if $p^{\prime}<p$ then the definition of interlacing pairs forces $\lambda_{2}\left(h_{p}\right)=1=\lambda_{1}\left(h_{p^{\prime}}\right)$. Set

$$
\beta_{\lambda}=\beta_{p^{\prime}, p+1}=\alpha_{p^{\prime}}+\cdots+\alpha_{p}+2\left(\alpha_{p+1}+\cdots+\alpha_{n-2}\right)+\alpha_{n-1}+\alpha_{n}
$$

and note that $\lambda_{1}\left(h_{\beta_{\lambda}}\right)=3-\delta_{p^{\prime}, p}$ and $\lambda_{2}\left(h_{\beta_{\lambda}}\right)=\left(1-\delta_{p^{\prime} p}\right)$. It is helpful to note that the assumption $\lambda\left(h_{n-1}+h_{n}\right) \in\{0,2\}$ implies that $p=n-2$ iff $\lambda\left(h_{n-1}+h_{n}\right)=2$.

Lemma. Suppose that $\lambda\left(h_{n-1}+h_{n}\right) \in\{0,2\}$ and $\lambda \neq \omega_{i-1}+\omega_{i}+\delta_{i+1, n} \omega_{n}$. Then $\lambda_{1}-\beta_{\lambda} \in P^{+}$ and there exists $\nu_{0} \in P^{+}$such that $\left(\lambda_{1}-\beta_{\lambda}-\nu_{0}, \lambda_{2}-\nu_{0}\right)$ is an interlacing pair.

Proof. It is simple to see that with our assumptions

$$
\lambda_{1}-\beta_{\lambda}=\lambda_{1}-\omega_{p+1}-\delta_{p, n-2} \omega_{n}-\left(1-\delta_{p^{\prime}, p}\right) \omega_{p^{\prime}}+\omega_{p^{\prime}-1}+\left(1-\delta_{p^{\prime}, p}\right) \omega_{p} \in P^{+} .
$$

Taking $\nu_{0}=\lambda_{2}\left(h_{p^{\prime}-1}\right) \omega_{p^{\prime}-1}+\left(1-\delta_{p^{\prime}, p}\right) \lambda_{2}\left(h_{p}\right) \omega_{p}$ it is easy to check that $\left(\lambda_{1}-\beta_{\lambda}-\nu_{0}, \lambda_{2}-\nu_{0}\right)$ is an interlacing pair. 
1.12. The second reduction is the following proposition which in particular, gives an upper bound for the dimension of $V\left(\lambda_{1}+\nu, \lambda_{2}+\nu\right)$.

Proposition. Suppose that $\lambda\left(h_{n-1}+h_{n}\right) \in\{0,2\}$ and $\lambda \neq \omega_{i-1}+\omega_{i}+\delta_{i+1, n} \omega_{n}$. There exists a right exact sequence of $\mathfrak{g}[t]$-modules

$$
\tau_{\left(\lambda_{1}+\nu\right)\left(h_{\beta_{\lambda}}\right)-1}^{*} V\left(\lambda_{1}+\nu-\beta_{\lambda}, \lambda_{2}+\nu\right) \rightarrow V\left(\lambda_{1}+\nu, \lambda_{2}+\nu\right) \rightarrow D(2, \lambda+2 \nu) \rightarrow 0,
$$

with $w_{\lambda_{1}+\nu-\beta_{\lambda}, \lambda_{2}+\nu} \rightarrow\left(x_{\beta_{\lambda}}^{-} \otimes t^{\left(\lambda_{1}+\nu\right)\left(h_{\beta_{\lambda}}\right)-1}\right) w_{\lambda_{1}+\nu, \lambda_{2}+\nu}$.

1.13. Assuming Proposition 1.10 and Proposition 1.12 we complete the proof of Theorem 1.9. The proof is by an induction with respect to the partial order on $P^{+}$. The minimal elements with respect to this order are $0, \omega_{1}, \omega_{n-1}, \omega_{n}$ and Proposition 1.10 shows that induction begins. Moreover the proposition also establishes the theorem if $\lambda=\omega_{i-1}+\omega_{i}+\delta_{i+1, n} \omega_{n}$ or $\lambda\left(h_{n-1}+h_{n}\right)=1$. Hence we have only to prove the inductive step when $\lambda\left(h_{n-1}+h_{n}\right) \in\{0,2\}$ and $\lambda \neq \omega_{i-1}+\omega_{i}+\delta_{i+1, n} \omega_{n}$. We shall need the following result to complete the proof of the inductive step.

Lemma. If $\lambda\left(h_{n-1}+h_{n}\right) \in\{0,2\}$ and $\lambda \neq \omega_{i-1}+\omega_{i}+\delta_{i+1, n} \omega_{n}$, there exists an inclusion of $\mathfrak{g}[t]$-modules

$$
\tau_{\left(\lambda_{1}+\nu\right)\left(h_{\beta_{\lambda}}\right)-1}^{*} D\left(1, \lambda_{1}+\nu-\beta_{\lambda}\right) \hookrightarrow D\left(1, \lambda_{1}+\nu\right),
$$

which sends to $w_{\lambda_{1}+\nu-\beta_{\lambda}} \rightarrow\left(x_{\beta_{\lambda}}^{-} \otimes t^{\left(\lambda_{1}+\nu\right)\left(h_{\beta_{\lambda}}\right)-1}\right) w_{\lambda_{1}+\nu}$.

Proof. By Lemma 1.5 it suffices to prove that $w:=\left(x_{\beta_{\lambda}}^{-} \otimes t^{\left(\lambda_{1}+\nu\right)\left(h_{\beta_{\lambda}}\right)-1}\right) w_{\lambda_{1}+\nu}$ satisfies the relations in (1.1). If $\beta_{\lambda}-\alpha_{i} \notin R^{+}$then it is clear that $\left(x_{i}^{+} \otimes t^{r}\right) w=0$ for all $r \in \mathbb{Z}_{+}$. Otherwise we must have $i=p+1$, or $i=p^{\prime}$ if $p^{\prime}<p$ or $i=n$ if $p=n-2$ and we have to prove that

$$
\left(x_{\beta_{\lambda}-\alpha_{i}}^{-} \otimes t^{\left(\lambda_{1}+\nu\right)\left(h_{\beta_{\lambda}}\right)-1+r}\right) w_{\lambda_{1}+\nu}=0, \quad r \in \mathbb{Z}_{+} .
$$

But this follows from (1.2) since in all cases $\lambda_{1}\left(h_{i}\right)=1$ and so $\left(\lambda_{1}+\nu\right)\left(h_{\beta_{\lambda}}\right)-1 \geq\left(\lambda_{1}+\right.$ $\nu)\left(h_{\beta_{\lambda}-\alpha_{i}}\right)$. The second relation in (1.1) is trivial if $r=0$ and if $r>0$ then it follows from (1.2) since

$$
\left(h \otimes t^{r}\right) w=\left(x_{\beta_{\lambda}}^{-} \otimes t^{\left(\lambda_{1}+\nu\right)\left(h_{\beta_{\lambda}}\right)+r-1}\right) w_{\lambda_{1}+\nu}=0 .
$$

The third relation is immediate since the modules are all finite-dimensional.

1.14. Lemma 1.6. Proposition 1.12 and the inductive hypothesis establish the following inequalities:

$$
\begin{gathered}
\operatorname{dim} D\left(\lambda_{1}+\nu, \lambda_{2}+\nu\right) \leq \operatorname{dim} V\left(\lambda_{1}+\nu, \lambda_{2}+\nu\right), \\
\operatorname{dim} V\left(\lambda_{1}+\nu, \lambda_{2}+\nu\right) \leq \operatorname{dim} D(2,2 \nu+\lambda)+\operatorname{dim} D\left(\lambda_{1}+\nu-\beta_{\lambda}, \lambda_{2}+\nu\right) .
\end{gathered}
$$

The inductive step follows if we prove that

$$
\operatorname{dim} D\left(\lambda_{1}+\nu, \lambda_{2}+\nu\right)=\operatorname{dim} D(2,2 \nu+\lambda)+\operatorname{dim} D\left(\lambda_{1}+\nu-\beta_{\lambda}, \lambda_{2}+\nu\right) .
$$

For this, we observe that Lemma 1.13 gives an inclusion

$$
0 \rightarrow D\left(1, \lambda_{1}+\nu-\beta_{\lambda}\right) \otimes D\left(1, \lambda_{2}+\nu\right) \rightarrow D\left(1, \lambda_{1}+\nu\right) \otimes D\left(\lambda_{2}+\nu\right),
$$


which sends

$$
w_{\lambda_{1}+\nu-\beta_{\lambda}} \otimes w_{\lambda_{2}+\nu} \rightarrow\left(\left(x_{\beta_{\lambda}}^{-} \otimes t^{\left.\left(\lambda_{1}+\nu\right)\left(h_{\beta_{\lambda}}\right)-1\right)}\right) w_{\lambda_{1}+\nu}\right) \otimes w_{\lambda_{2}+\nu} .
$$

Since $\left(\lambda_{1}+\nu\right)\left(h_{\beta_{\lambda}}\right)-1 \geq\left(\lambda_{2}+\nu\right)\left(h_{\beta_{\lambda}}\right)$ we see that (1.2) gives

$$
\left(x_{\beta_{\lambda}}^{-} \otimes t^{\left.\left(\lambda_{1}+\nu\right)\left(h_{\beta_{\lambda}}\right)-1\right)}\right)\left(w_{\lambda_{1}+\nu} \otimes w_{\lambda_{2}+\nu}\right)=\left(\left(x_{\beta_{\lambda}}^{-} \otimes t^{\left(\lambda_{1}+\nu\right)\left(h_{\beta_{\lambda}}\right)-1}\right) w_{\lambda_{1}+\nu}\right) \otimes w_{\lambda_{2}+\nu} .
$$

It follows that we have an inclusion

$$
\iota: D\left(\lambda_{1}+\nu-\beta_{\lambda}, \lambda_{2}+\nu\right) \hookrightarrow D\left(\lambda_{1}+\nu, \lambda_{2}+\nu\right)
$$

and it suffices to prove that the corresponding quotient is isomorphic to $D(2,2 \nu+\lambda)$. By Lemma 1.7 we have the following surjective maps

$$
V\left(\lambda_{1}+\nu, \lambda_{2}+\nu\right) \rightarrow D\left(\lambda_{1}+\nu, \lambda_{2}+\nu\right) \rightarrow D(2,2 \nu+\lambda) .
$$

These maps are all unique up to scalars and Proposition 1.12 shows that the kernel of the composite map is generated by the element $\left(x_{\beta_{\lambda}}^{-} \otimes t^{\left.\left(\lambda_{1}+\nu\right)\left(h_{\beta_{\lambda}}\right)-1\right)}\right) w_{\lambda_{1}+\nu, \lambda_{2}+\nu}$. Hence the kernel of

$$
D\left(\lambda_{1}+\nu, \lambda_{2}+\nu\right) \rightarrow D(2,2 \nu+\lambda)
$$

is generated by $\left(x_{\beta_{\lambda}}^{-} \otimes t^{\left.\left(\lambda_{1}+\nu\right)\left(h_{\beta_{\lambda}}\right)-1\right)}\right)\left(w_{\lambda_{1}+\nu} \otimes w_{\lambda_{2}+\nu}\right)$. But this means that the latter kernel is precisely the image of $\iota$ and hence the corresponding quotient is isomorphic to $D(2,2 \nu+\lambda)$ as needed.

1.15. Given a graded $\mathfrak{g}[t]$-module $V=\oplus_{s \in \mathbb{Z}} V[s]$ we have,

$$
\left(\mathfrak{g} \otimes t^{r}\right) V[s] \subset V[r+s], \quad V_{\mu}=\bigoplus_{s \in \mathbb{Z}} V_{\mu}[s], \quad V_{\mu}[s]=V_{\mu} \cap V[s],
$$

for all $r, s \in \mathbb{Z}$ and $\mu \in P$. Let $\mathbb{Z}\left[v, v^{-1}\right][P]$ be the group ring of $P$ with coefficients in $\mathbb{Z}\left[v, v^{-1}\right]$ where $v$ is an indeterminate. Let $e(\mu), \mu \in P$ be a basis for the group ring and set

$$
\operatorname{ch}_{\mathrm{gr}} V=\sum_{\mu \in P}\left(\sum_{s \in \mathbb{Z}} \operatorname{dim} V_{\mu}[s] v^{s}\right) e(\mu) .
$$

Notice that in the course of proving Theorem 1.9 we also established that the right exact sequence in Proposition 1.12 is exact. Hence we get an equality of graded characters

$$
\operatorname{ch}_{\mathrm{gr}} D\left(\lambda_{1}+\nu, \lambda_{2}+\nu\right)=\operatorname{ch}_{\mathrm{gr}} D(2,2 \nu+\lambda)+v^{\left(\lambda_{1}+\nu\right)\left(h_{\beta_{\lambda}}\right)-1} \operatorname{ch}_{\mathrm{gr}} D\left(\lambda_{1}-\beta_{\lambda}+\nu, \lambda_{2}+\nu\right) \text {. }
$$

Using this we can derive a formula for $\operatorname{ch}_{\mathrm{gr}} D\left(\lambda_{1}+\nu, \lambda_{2}+\nu\right)$ in terms of Demazure modules as follows.

Given $\mu \in P^{+}$define elements $\lfloor\mu / 2\rfloor$ and $\operatorname{res}_{2} \mu$ of $P^{+}$by,

$$
\lfloor\mu / 2\rfloor\left(h_{i}\right)=\left\lfloor\mu\left(h_{i}\right) / 2\right\rfloor, \quad 1 \leq i \leq n, \quad \operatorname{res}_{2} \mu=\mu-2\lfloor\mu / 2\rfloor .
$$

Then $\operatorname{res}_{2} \mu \in P^{+}(1)$ and we set $\beta_{\mu}=\beta_{\operatorname{res}_{2} \mu}$ if $\operatorname{res}_{2} \mu$ satisfies the conditions of Section 1.11 and otherwise set $\beta_{\mu}=0$. Let $\left(\mu_{1}, \mu_{2}\right)$ be the interlacing pair corresponding to $\operatorname{res}_{2} \mu$ and let

$$
r_{\mu}=\lfloor\mu / 2\rfloor\left(h_{\beta_{\mu}}\right)+\max \left\{\mu_{1}\left(h_{\beta_{\mu}}\right), \mu_{2}\left(h_{\beta_{\mu}}\right)\right\}-1 \text {. }
$$

Given $\mu \in P^{+}$set

$$
\mu^{0}=\mu, \quad \mu^{1}=\mu^{0}-\beta_{\mu^{0}}, \ldots, \mu^{s}=\mu^{s-1}-\beta_{\mu^{s-1}},
$$


where $s$ is minimal so that $\beta_{\mu^{s}}=0$. Setting $r_{k}=r_{\mu^{k}}$ a repeated application of (1.8) gives

$$
\operatorname{ch}_{\mathrm{gr}} D\left(\lfloor\mu / 2\rfloor+\mu_{1},\lfloor\mu / 2\rfloor+\mu_{2}\right)=\sum_{k=0}^{s} v^{r_{k}} \operatorname{ch}_{\mathrm{gr}} D\left(2, \mu^{k}\right) \text {. }
$$

\section{Proof of Proposition 1.10 and Proposition 1.12.}

In this section we first establish some technical results on the properties of interlacing pairs and then prove Proposition 1.10 and Proposition 1.12 .

Throughout this section we shall assume without further mention that $\left(\lambda_{1}, \lambda_{2}\right)$ is an interlacing pair and $\lambda=\lambda_{1}+\lambda_{2}$. We shall also assume without loss of generality that there exists $p$ maximal with $\lambda\left(h_{p+1}\right)=1$ with $p \leq n-2$ and $\lambda_{1}\left(h_{p+1}\right)=1$.

\section{1.}

Lemma. For all $1 \leq i \leq j \leq n$ we have

$$
\left|\left(\lambda_{1}-\lambda_{2}\right)\left(h_{i, j}\right)\right| \leq 1
$$

and hence $\left|\left(\lambda_{1}-\lambda_{2}\right)\left(h_{\alpha}\right)\right| \leq 2$ for all $\alpha \in R^{+}$. Further if $\lambda\left(h_{n-1}+h_{n}\right)=1$ then

$$
\left|\left(\lambda_{1}-\lambda_{2}\right)\left(h_{\alpha}\right)\right| \leq 1 \text { for all } \alpha \in R^{+} \text {. }
$$

Proof. Equation (2.1) is proved by induction on $j-i$; induction obviously begins at $i=j$ since $\lambda \in P^{+}(1)$. If $j>i$ the inductive step is clear if $\lambda\left(h_{i}\right)=0$ or $\lambda\left(h_{j}\right)=0$. If $\lambda\left(h_{i}\right)=1=\lambda\left(h_{j}\right)$ then either $\lambda\left(h_{s}\right)=0$ for all $i<s<j$ or there exists $i<p<j$ minimal such that $\lambda_{2}\left(h_{p}\right)=1$. In the first case we have proved that $\left(\lambda_{1}-\lambda_{2}\right)\left(h_{i, j}\right)=0$ and in the second case we have proved that $\left(\lambda_{1}-\lambda_{2}\right)\left(h_{i, j}\right)=\left(\lambda_{1}-\lambda_{2}\right)\left(h_{p+1, j}\right)$ and the inductive step follows. Since $\beta_{i, j}=\alpha_{i, n}+\alpha_{j, n-1}$ for $1 \leq i<j \leq n-1$ it is immediate that

$$
\left|\left(\lambda_{1}-\lambda_{2}\right)\left(\beta_{i, j}\right)\right| \leq 2 \text { for all } 1 \leq i<j \leq n-1 .
$$

Moreover, if $\lambda\left(h_{n-1}+h_{n}\right)=1$ our assumptions imply that $\lambda_{1}\left(h_{n-1}\right)=1$ and $\lambda_{2}\left(h_{n-1}+h_{n}\right)=0$ and so using (2.1) we have

$$
\begin{gathered}
-1 \leq\left(\lambda_{1}-\lambda_{2}\right)\left(h_{i, n}\right)=\left(\lambda_{1}-\lambda_{2}\right)\left(h_{i, n-2}\right)=\left(\lambda_{1}-\lambda_{2}\right)\left(h_{i, n-1}\right)-1 \leq 0, \\
1 \geq\left(\lambda_{1}-\lambda_{2}\right)\left(h_{j, n-1}\right)=\left(\lambda_{1}-\lambda_{2}\right)\left(h_{j, n-2}\right)+1 \geq 0 .
\end{gathered}
$$

Hence we have

$$
-1 \leq\left(\lambda_{1}-\lambda_{2}\right)\left(h_{\beta_{i, j}}\right)=\left(\lambda_{1}-\lambda_{2}\right)\left(h_{i, n}\right)+\left(\lambda_{1}-\lambda_{2}\right)\left(h_{j, n-1}\right) \leq 1
$$

and the proof of the lemma is complete.

2.2. Recall our convention that $\alpha_{i, j}=0$ if $i>j$. Set

$$
R\left(\lambda_{1}, \lambda_{2}\right)=\left\{\beta_{i, j}:\left(\lambda_{1}-\lambda_{2}\right)\left(h_{\beta_{i, j}}\right)= \pm 2\right\} .
$$

Note that Lemma 2.1 shows that $R\left(\lambda_{1}, \lambda_{2}\right)=\emptyset$ if $\lambda\left(h_{n-1}+h_{n}\right)=1$ or $\lambda=\omega_{i-1}+\omega_{i}+\delta_{i, n-1} \omega_{n}$. The next result establishes the converse.

Lemma. Suppose that $\lambda\left(h_{n-1}+h_{n}\right) \in\{0,2\}$ and $\lambda \neq \omega_{i-1}+\omega_{i}+\delta_{i, n-1} \omega_{n}$. Then $\beta_{\lambda} \in R\left(\lambda_{1}, \lambda_{2}\right)$ and more generally,

$\beta_{i, j} \in R\left(\lambda_{1}, \lambda_{2}\right) \Longleftrightarrow \beta_{i, j}=\alpha_{i, p^{\prime}-1}+\beta_{\lambda}+\alpha_{j, p}$ and $\left(\lambda_{1}-\lambda_{2}\right)\left(h_{i, p^{\prime}-1}\right)=0=\left(\lambda_{1}-\lambda_{2}\right)\left(h_{j, p}\right)=0$. 
Proof. Recall from Section 1.11 that $\beta_{\lambda}=\beta_{p^{\prime}, p+1}$ where $1 \leq p^{\prime} \leq p$ is maximal with $\left(\lambda_{1}-\right.$ $\left.\lambda_{2}\right)\left(h_{p^{\prime}, p}\right)=0$ and that $\left(\lambda_{1}-\lambda_{2}\right)\left(h_{\beta_{\lambda}}\right)=2$. Hence a calculation shows that

$$
\beta_{i, j} \in R\left(\lambda_{1}, \lambda_{2}\right) \Longrightarrow i \leq p^{\prime} \text { or } p^{\prime}<j \leq p+1 \text {, }
$$

which shows that $\beta_{i, j}=\alpha_{i, p^{\prime}-1}+\beta_{\lambda}+\alpha_{j, p}$.

Since $\beta_{i, p+1} \in R^{+}$if $i<p^{\prime}$ we have

$$
\left(\lambda_{1}-\lambda_{2}\right)\left(h_{\beta_{i, p+1}}\right)=\left(\lambda_{1}-\lambda_{2}\right)\left(h_{i, p^{\prime}-1}\right)+2
$$

and Lemma 2.1 forces $\left(\lambda_{1}-\lambda_{2}\right)\left(h_{i, p^{\prime}-1}\right) \in\{-1,0\}$. If $j<p+1$ we have $\beta_{p^{\prime}, j}=\beta_{p^{\prime}, p+1}+\alpha_{j, p}$ and hence a similar argument shows that $\left(\lambda_{1}-\lambda_{2}\right)\left(h_{j, p}\right) \in\{-1,0\}$. Together with hypothesis that $\left(\lambda_{1}-\lambda_{2}\right)\left(h_{\beta_{i, j}}\right)= \pm 2$ we get $\left(\lambda_{1}-\lambda_{2}\right)\left(h_{i, p^{\prime}-1}\right)=0=\left(\lambda_{1}-\lambda_{2}\right)\left(h_{j, p}\right)$ as needed. The converse is obvious.

Remark. Notice that in particular we have established (with our conventions on $\left(\lambda_{1}, \lambda_{2}\right)$ ) that $\beta \in R\left(\lambda_{1}, \lambda_{2}\right)$ iff $\left(\lambda_{1}-\lambda_{2}\right)\left(h_{\beta}\right)=2$.

2.3. Assume that $\lambda\left(h_{n-1}+h_{n}\right)=1$ or that $\lambda=\omega_{i-1}+\omega_{i}+\delta_{i, n-1} \omega_{n}$. By Lemma 2.1 we have $\left(\lambda_{1}-\lambda_{2}\right)\left(h_{\alpha}\right) \in\{-1,0,1\}$ for all $\alpha \in R^{+}$, and so

$$
\max \left\{\left(\nu+\lambda_{1}\right)\left(h_{\alpha}\right),\left(\nu+\lambda_{2}\right)\left(h_{\alpha}\right)\right\}=\nu\left(h_{\alpha}\right)+\left\lceil\left(\lambda\left(h_{\alpha}\right) / 2\right\rceil .\right.
$$

The defining relations give

$$
V\left(\lambda_{1}+\nu, \lambda_{2}+\nu\right) \cong D\left(2,2 \nu+\lambda_{1}+\lambda_{2}\right) .
$$

Since the maps in Lemma 1.6 and Lemma 1.7 are unique up to scalars it now follows that the map

$$
V\left(\lambda_{1}+\nu, \lambda_{2}+\nu\right) \rightarrow D\left(\lambda_{1}+\nu, \lambda_{2}+\nu\right) \rightarrow D\left(2,2 \nu+\lambda_{1}+\lambda_{2}\right),
$$

is an isomorphism and hence all the maps are isomorphisms. Proposition 1.10 is proved.

2.4. We turn to the proof of Proposition 1.12, We have $R\left(\lambda_{1}, \lambda_{2}\right) \neq \emptyset$ and our conventions imply that $\lambda_{1}\left(h_{\beta_{\lambda}}\right)=3-\delta_{p^{\prime}, p}$ and $\lambda_{2}\left(h_{\beta_{\lambda}}\right)=1-\delta_{p^{\prime}, p}$. Using the remark following Lemma 2.2 we have $\lambda_{1}\left(h_{\beta}\right) \geq \lambda_{2}\left(h_{\beta}\right)$ for all $\beta \in R\left(\lambda_{1}, \lambda_{2}\right)$. Hence for all $\beta \in R\left(\lambda_{1}, \lambda_{2}\right)$,

$$
\left(\nu+\lambda_{1}\right)\left(h_{\beta}\right)=\max \left\{\left(\nu+\lambda_{1}\right)\left(h_{\beta}\right),\left(\nu+\lambda_{2}\right)\left(h_{\beta}\right)\right\}=\nu\left(h_{\beta}\right)+\left\lceil\left(\lambda\left(h_{\beta}\right) / 2\right\rceil+1, .\right.
$$

An inspection of the defining relations of the modules shows that the kernel $K$ of the canonical map

$$
\psi_{\lambda_{1}+\nu, \lambda_{2}+\nu}: V\left(\lambda_{1}+\nu, \lambda_{2}+\nu\right) \rightarrow D(2, \lambda+\nu) \rightarrow 0, \quad w_{\lambda_{1}+\nu, \lambda_{2}+\nu} \rightarrow w_{2 \nu+\lambda},
$$

is generated by the elements

$$
\left(x_{\beta}^{-} \otimes t^{\left(\nu+\lambda_{1}\right)\left(h_{\beta}\right)-1}\right) w_{\lambda_{1}+\nu, \lambda_{2}+\nu}, \quad \beta \in R\left(\lambda_{1}, \lambda_{2}\right) .
$$

The proof of Proposition 1.12 is now shown in two steps. The first step is to show that

$$
K=\mathbb{U}(\mathfrak{g}[t])\left(x_{\beta_{\lambda}}^{-} \otimes t^{\left.\left(\nu+\lambda_{1}\right)\left(h_{\beta_{\lambda}}\right)-1\right)}\right) w_{\lambda_{1}+\nu, \lambda_{2}+\nu} .
$$

For this, let $\beta \in R\left(\lambda_{1}, \lambda_{2}\right)$ and write $\beta=\alpha_{i, p^{\prime}-1}+\beta_{p^{\prime}, p+1}+\alpha_{j, p}$ as in Lemma 2.2 and assume that $i \leq p^{\prime}-1$ or $j \leq p$ (otherwise $\beta=\beta_{\lambda}$ and there is nothing to prove). The defining relations

$$
\left(x_{i, p^{\prime}-1}^{-} \otimes t^{\left(\nu+\lambda_{1}\right)\left(h_{i, p^{\prime}-1}\right)}\right) w_{\lambda_{1}+\nu, \lambda_{2}+\nu}=0=\left(x_{j, p}^{-} \otimes t^{\left(\nu+\lambda_{1}\right)\left(h_{j, p}\right)}\right) w_{\lambda_{1}+\nu, \lambda_{2}+\nu}
$$


imply if $i \leq p^{\prime}-1$ and $j \leq p$

$$
\begin{gathered}
\left(x_{i, p^{\prime}-1}^{-} \otimes t^{\left(\nu+\lambda_{1}\right)\left(h_{i, p^{\prime}-1}\right)}\right)\left(x_{j, p}^{-} \otimes t^{\left(\nu+\lambda_{1}\right)\left(h_{j, p}\right)}\right)\left(x_{\beta_{\lambda}}^{-} \otimes t^{\left(\nu+\lambda_{1}\right)\left(h_{\beta_{\lambda}}\right)-1}\right) w_{\lambda_{1}+\nu, \lambda_{2}+\nu}= \\
\left.\left[x_{i, p^{\prime}-1}^{-},\left[x_{j, p}^{-}, x_{\beta_{\lambda}}^{-}\right]\right] \otimes t^{\left(\nu+\lambda_{1}\right)\left(h_{\beta_{i, j}}\right)-1}\right) w_{\lambda_{1}+\nu, \lambda_{2}+\nu}=\left(x_{\beta_{i, j}}^{-} \otimes t^{\left(\nu+\lambda_{1}\right)\left(h_{\beta_{i, j}}\right)-1}\right) w_{\lambda_{1}+\nu, \lambda_{2}+\nu} .
\end{gathered}
$$

An obvious modification works if $i \geq p^{\prime}$ or $j \geq p+1$ and the first step is proved.

The next step is to show that the element $\left(x_{\beta_{\lambda}}^{-} \otimes t^{\left(\nu+\lambda_{1}\right)\left(h_{\beta_{\lambda}}\right)-1}\right) w_{\lambda_{1}+\nu, \lambda_{2}+\nu}$ satisfies the defining relations of the element $w_{\lambda_{1}+\nu-\beta_{\lambda}, \lambda_{2}+\nu} \in V\left(\lambda_{1}+\nu-\beta_{\lambda}, \lambda_{2}+\nu\right)$. This establishes the existence of the map $V\left(\lambda_{1}+\nu-\beta_{\lambda}, \lambda_{2}+\nu\right) \rightarrow K \rightarrow 0$.

2.5. We prove that $\left(x_{\beta_{\lambda}}^{-} \otimes t^{\left(\nu+\lambda_{1}\right)\left(h_{\beta_{\lambda}}\right)-1}\right) w_{\lambda_{1}+\nu, \lambda_{2}+\nu}$ satisfies the relations in (1.3) with $\left(\lambda_{1}, \lambda_{2}\right)$ replaced by $\left(\lambda_{1}-\beta_{\lambda}, \lambda_{2}\right)$. Since $\left(x_{i}^{+} \otimes t^{r}\right) w_{\lambda_{1}+\nu, \lambda_{2}+\nu}=0$ for $1 \leq i \leq n$ and $r \in \mathbb{Z}_{+}$the first relation in (1.3) is immediate if $\beta_{\lambda}-\alpha_{i} \notin R^{+}$. Otherwise we must prove that

$$
\text { (†) }\left(x_{\beta_{\lambda}-\alpha_{i}}^{-} \otimes t^{\left(\nu+\lambda_{1}\right)\left(h_{\beta_{\lambda}}\right)-1+r}\right) w_{\lambda_{1}+\nu, \lambda_{2}+\nu}=0 \text {. }
$$

Since $\beta_{\lambda}=\beta_{p^{\prime}, p+1}$ it follows that either $i=p+1$ and if $p^{\prime}<p$ then we can also have $i=p^{\prime}$ and if $p=n-2$ then, in addition we can have $i=n$. In the first two cases we have $\lambda_{1}\left(h_{i}\right)=1$ and $\lambda_{2}\left(h_{i}\right)=0$ (see Section 1.11). In particular

$$
\left(x_{\beta_{\lambda}-\alpha_{i}}^{-} \otimes t^{\nu\left(h_{\beta_{\lambda}-\alpha_{i}}\right)+2-\delta_{p, p^{\prime}}}\right) w_{\lambda_{1}+\nu, \lambda_{2}+\nu}=0
$$

is a defining relation and $(\dagger)$ follows by applying $\left(h \otimes t^{r}\right)$ since

$$
\left(\nu+\lambda_{1}\right)\left(h_{\beta_{\lambda}}\right)-1+r=\nu\left(h_{\beta_{\lambda}}\right)+2-\delta_{p, p^{\prime}}+r \geq \nu\left(h_{\beta_{\lambda}-\alpha_{i}}\right)+2-\delta_{p, p^{\prime}}, \quad r \in \mathbb{Z}_{+} .
$$

A similar argument establishes the case when $p=n-2$ and $i=n$.

It is trivial to see that the second relation in (1.1) holds and the third is then immediate since $V\left(\nu+\lambda_{1}, \nu+\lambda_{2}\right)$ is finite-dimensional.

2.6. We need the following result to prove that $\left(x_{\beta_{\lambda}}^{-} \otimes t^{\left(\nu+\lambda_{1}\right)\left(h_{\beta_{\lambda}}\right)-1}\right) w_{\lambda_{1}+\nu, \lambda_{2}+\nu}$ satisfies the relations in (1.4).

Lemma. The relation $\left(x_{\alpha}^{-} \otimes t^{\nu\left(h_{\alpha}\right)+\max \left\{\lambda_{1}\left(h_{\alpha}\right), \lambda_{2}\left(h_{\alpha}\right)\right\}}\right) w_{\lambda_{1}+\nu, \lambda_{2}+\nu}=0$ holds in $V\left(\lambda_{1}+\nu, \lambda_{2}+\nu\right)$ for all $\alpha \in R^{+}$iff it holds for all the elements of the set

$$
\left\{\alpha_{i, j}: 1 \leq i \leq j \leq n, \quad \lambda\left(h_{i}\right)=1=\lambda\left(h_{j}\right), \quad \lambda\left(h_{i, j}\right)=2-\delta_{i, j}\right\} .
$$

Proof. The forward direction of the Lemma is obvious. For the reverse direction we proceed by induction with respect to the partial order on $R^{+}$induced by the partial order $\leq$on $P$. If $\alpha=\alpha_{i}$ for some $1 \leq i \leq n$ there is nothing to prove. For the inductive step choose $1 \leq r \leq n$ so that $\alpha-\alpha_{r} \in R^{+}$. If $\lambda\left(h_{r}\right)=0$ then the result follows from $\left[x_{r}^{-}, x_{\alpha-\alpha_{r}}^{-}\right]=x_{\alpha}^{-}$and

$$
\left(x_{r}^{-} \otimes t^{\nu\left(h_{r}\right)}\right) w_{\lambda_{1}+\nu, \lambda_{2}+\nu}=0=\left(x_{\alpha-\alpha_{r}}^{-} \otimes t^{\nu\left(h_{\alpha-\alpha_{r}}\right)+\max \left\{\lambda_{1}\left(h_{\alpha}\right), \lambda_{2}\left(h_{\alpha}\right)\right\}}\right) w_{\lambda_{1}+\nu, \lambda_{2}+\nu},
$$

where the second equality is an application of the inductive hypothesis. Otherwise, $\lambda\left(h_{r}\right)>0$ for all $1 \leq r \leq n$ with $\alpha-\alpha_{r} \in R^{+}$, in which case $\alpha$ is one of the following:

- $\alpha_{i, j}$ with $\lambda\left(h_{i}\right)=1=\lambda\left(h_{j}\right)$,

- $\beta_{i, j}$ with $i<j-1$ and $\lambda\left(h_{i}\right)=\lambda\left(h_{j}\right)=1$,

- $\beta_{j-1, j}$ with $\lambda\left(h_{j}\right)=1$. 
In the first two cases choose $i<s \leq j$ minimal with $\lambda\left(h_{s}\right)=1$ and $\lambda\left(h_{i, s}\right)=2$. The result follows since Lemma 2.1 and the inductive hypothesis give

$$
\left(x_{i, s}^{-} \otimes t^{\nu\left(h_{i, s}\right)+1}\right) w_{\lambda_{1}+\nu, \lambda_{2}+\nu}=0=\left(x_{\alpha-\alpha_{i, s}}^{-} \otimes t^{\nu\left(h_{\alpha-\alpha_{i, s}}\right)+\max \left\{\lambda_{1}\left(h_{\alpha}\right)-1, \lambda_{2}\left(h_{\alpha}\right)-1\right\}}\right) w_{\lambda_{1}+\nu, \lambda_{2}+\nu} .
$$

In the third case if $\lambda\left(h_{j-1}\right)=1$ or if $\lambda\left(h_{j-1}\right)=0$ and there exists $s>j$ minimal with $\lambda\left(h_{s}\right)=1$ then the result follows as before by working with the pairs $\left(\alpha_{j-1, j}, \alpha-\alpha_{j-1, j}\right)$ and $\left(\alpha_{j-1, s}, \alpha-\alpha_{j-1, s}\right)$ respectively. Otherwise we have $\max \left\{\lambda_{1}\left(h_{\alpha}\right), \lambda_{2}\left(h_{\alpha}\right)\right\}=2$ and the result follows from

$$
\left(x_{j-1, n-1}^{-} \otimes t^{\nu\left(h_{j-1, n-1}\right)+1}\right) w_{\lambda_{1}+\nu, \lambda_{2}+\nu}=0=\left(x_{j, n}^{-} \otimes t^{\nu\left(h_{j, n}\right)+1}\right) w_{\lambda_{1}+\nu, \lambda_{2}+\nu} .
$$

2.7. To complete the proof of the second step, we now show that (1.4) also holds. By the preceding Lemma it suffices to prove that

$$
(*) \quad\left(x_{\alpha}^{-} \otimes t^{\nu\left(h_{\alpha}\right)+\max \left\{\left(\lambda_{1}-\beta_{\lambda}\right)\left(h_{\alpha}\right), \lambda_{2}\left(h_{\alpha}\right)\right\}}\right)\left(x_{\beta_{\lambda}}^{-} \otimes t^{\left(\nu+\lambda_{1}\right)\left(h_{\beta_{\lambda}}\right)-1}\right) w_{\lambda_{1}+\nu, \lambda_{2}+\nu}=0
$$

for roots of the form $\alpha=\alpha_{i, j}$ with

$$
(* *) \quad\left(\lambda-\beta_{\lambda}\right)\left(h_{i}\right)=1=\left(\lambda-\beta_{\lambda}\right)\left(h_{j}\right), \quad\left(\lambda-\beta_{\lambda}\right)\left(h_{s}\right)=0, \quad i<s<j,
$$

and note that this forces $\beta_{\lambda}\left(h_{i}\right) \leq 0$ and $\beta_{\lambda}\left(h_{j}\right) \leq 0$. We claim that $\left(\beta_{\lambda}, \alpha\right) \leq 0$. Otherwise we would have that $\beta_{\lambda}-\alpha \in R$ and since $\alpha-\beta_{\lambda} \notin R^{+}$we must have $\beta_{\lambda}-\alpha \in R^{+}$. It is elementary to see that this is only possible if $\alpha_{i, j}=\alpha_{p^{\prime}, r}$ or $\alpha_{p+1, r}$ for some $r \neq p$ and $p^{\prime}<r \leq n$. Since $\beta_{p^{\prime}, p+1}\left(h_{p^{\prime}}\right)=1-\delta_{p^{\prime}, p}$, and $\beta_{p^{\prime}, p+1}\left(h_{p+1}\right)=1$ we see that $(* *)$ forces $p^{\prime}=p$ and $\alpha=\alpha_{p, r}$. However, if $p^{\prime}=p$ then we have $\lambda\left(h_{p}\right)=0$ by definition and $\beta_{\lambda}\left(h_{p}\right)=0$ which again contradicts $(* *)$ and the claim is proved.

If $\alpha+\beta_{\lambda} \notin R^{+}$, i.e. $\left(\alpha, \beta_{\lambda}\right)=0$ then

$$
\left(x_{\alpha}^{-} \otimes t^{\nu\left(h_{\alpha}\right)+\max \left\{\left(\lambda_{1}-\beta_{\lambda}\right)\left(h_{\alpha}\right), \lambda_{2}\left(h_{\alpha}\right)\right\}}\right) w_{\lambda_{1}+\nu, \lambda_{2}+\nu}=0,
$$

is a defining relation and $(*)$ follows since $\left[x_{\alpha}^{-}, x_{\beta_{\lambda}}^{-}\right]=0$. If $\left(\alpha, \beta_{\lambda}\right)<0$ then $\alpha+\beta_{\lambda} \in R^{+}$and $\left[x_{\alpha}^{-}, x_{\beta_{\lambda}}^{-}\right]=x_{\alpha+\beta_{\lambda}}^{-}$. It suffices to show that

$$
\left(x_{\alpha+\beta_{\lambda}}^{-} \otimes t^{\nu\left(h_{\alpha}\right)+\max \left\{\left(\lambda_{1}-\beta_{\lambda}\right)\left(h_{\alpha}\right), \lambda_{2}\left(h_{\alpha}\right)\right\}+\left(\nu+\lambda_{1}\right)\left(h_{\beta_{\lambda}}\right)-1}\right) w_{\lambda_{1}+\nu, \lambda_{2}+\nu}=0 .
$$

But this holds since

$$
\begin{aligned}
\max \left\{\lambda_{1}\left(h_{\alpha+\beta_{\lambda}}\right), \lambda_{2}\left(h_{\alpha+\beta_{\lambda}}\right)\right\} & =\max \left\{\lambda_{1}\left(h_{\alpha}\right)+3-\delta_{p^{\prime}, p}, \lambda_{2}\left(h_{\alpha}\right)+1-\delta_{p^{\prime}, p}\right\} \\
& \leq \max \left\{\lambda_{1}\left(h_{\alpha}\right)+3-\delta_{p^{\prime}, p}, \lambda_{2}\left(h_{\alpha}\right)+2-\delta_{p^{\prime}, p}\right\} \\
& =\max \left\{\lambda_{1}\left(h_{\alpha}\right)+1, \lambda_{2}\left(h_{\alpha}\right)\right\}+2-\delta_{p^{\prime}, p} .
\end{aligned}
$$

This completes the proof of Proposition 1.12.

\section{Connection with the Representation theory of Quantum Affine Algebras}

In this section we explain very briefly the relationship between our results and the representation theory of quantum affine algebras. We also discuss the connection with the papers [19, 20] on monoidal categorification. 
3.1. Let $\mathbb{C}(q)$ be the field of rational functions in an indeterminate $q$ and set $\mathbb{A}=\mathbb{Z}\left[q, q^{-1}\right]$. Let $\mathbb{U}_{q}(\widehat{\mathfrak{g}})$ be the quantized enveloping algebra (defined over $\left.\mathbb{C}(q)\right)$ associated to $\widehat{\mathfrak{g}}$. Let $\mathbb{U}_{\mathbb{A}}(\widehat{\mathfrak{g}})$ be the $\mathbb{A}$-form of $\mathbb{U}_{q}(\widehat{\mathfrak{g}})$ and recall that it is a free $\mathbb{A}$-module and

$$
\mathbb{U}_{q}(\widehat{\mathfrak{g}}) \cong \mathbb{U}_{\mathbb{A}}(\widehat{\mathfrak{g}}) \otimes_{\mathbb{A}} \mathbb{C}(q) .
$$

Regarding $\mathbb{C}$ as an $\mathbb{A}$-module by letting $q$ act as 1 we have that $\mathbb{U}_{\mathbb{A}}(\widehat{\mathfrak{g}}) \otimes_{\mathbb{A}} \mathbb{C}$ is an algebra over $\mathbb{C}$ which has the universal enveloping algebra $\mathbb{U}(\widehat{\mathfrak{g}})$ as a canonical quotient. Finally, recall that $\mathbb{U}_{q}(\widehat{\mathfrak{g}})$ is a Hopf algebra and that $\mathbb{U}_{\mathbb{A}}(\widehat{\mathfrak{g}})$ is a Hopf subalgebra.

Let $\mathcal{P}_{\mathbb{Z}}^{+}$be the free abelian monoid generated by elements $\left\{\boldsymbol{\omega}_{i, q^{r}}: 1 \leq i \leq n, r \in \mathbb{Z}\right\}$ and let wt : $\mathcal{P}^{+} \rightarrow P^{+}$be the morphism of monoids given by wt $\boldsymbol{\pi}=\sum_{i=1}^{n}\left(\operatorname{deg} \pi_{i}\right) \omega_{i}$.

Definition. Let $\mathcal{P}_{\mathbb{Z}}^{+}(1)$ be the subset of $\mathcal{P}_{\mathbb{Z}}^{+}$containing the identity of the and elements of the form $\boldsymbol{\omega}_{i_{1}, a_{1}} \cdots \boldsymbol{\omega}_{i_{k}, a_{k}}$, where $1 \leq i_{1}<i_{2}<\cdots<i_{k} \leq n, a_{j} \in q^{\mathbb{Z}}$ for $1 \leq k \leq n$, and

$$
\begin{gathered}
a_{j} / a_{j+1}=q^{ \pm\left(i_{j+1}-i_{j}+2\right)}, \quad k \geq 2, \\
a_{j} / a_{j+1}=q^{ \pm\left(i_{j+1}-i_{j}+2\right)} \Longrightarrow a_{j+1} / a_{j+2}=q^{\mp\left(i_{j+2}-i_{j+1}+2\right)}
\end{gathered}
$$

where the second requirement holds for all $j \leq k-2$ if $\left(i_{k-1}, i_{k}\right) \neq(n-1, n)$ and if $\left(i_{k-1}, i_{k}\right)=$ $(n-1, n)$ then it holds for $j \leq k-3$ and we require $a_{k-1}=a_{k}$.

Clearly wt $\mathcal{P}_{\mathbb{Z}}^{+}(1)=P^{+}(1)$.

3.2. To each element $\boldsymbol{\pi} \in \mathcal{P}_{\mathbb{Z}}^{+}$one can associate an (unique up to isomorphism) irreducible finite dimensional representation $[\boldsymbol{\pi}]$ of $\mathbb{U}_{q}(\widehat{\mathfrak{g}})$. The trivial representation corresponds to the identity of the monoid. Given $\boldsymbol{\pi}, \boldsymbol{\pi}^{\prime} \in \mathcal{P}_{\mathbb{Z}}^{+}(1)$, the tensor product $[\boldsymbol{\pi}] \otimes\left[\boldsymbol{\pi}^{\prime}\right]$ is generically irreducible and isomorphic to $\left[\boldsymbol{\pi} \boldsymbol{\pi}^{\prime}\right]$. However, necessary and sufficient conditions for this to hold are not known outside the case of $\mathfrak{s l}_{2}$ and motivates the interest in understanding the prime irreducible representations. Recall that a representation is prime if it cannot be written as a tensor product of two non-trivial representations. The following result is not hard to prove (see for instance [3, 4] for a similar statement for $A_{n}$ ).

Lemma. The module $[\boldsymbol{\pi}]$ is prime for all $\boldsymbol{\pi} \in \mathcal{P}_{\mathbb{Z}}^{+}(1)$.

The results of $\left[5,9\right.$, show that the representation $[\boldsymbol{\pi}]$ admits an $\mathbb{A}$-form denoted $\left[\boldsymbol{\pi}_{\mathbb{A}}\right]$ and $\left[\boldsymbol{\pi}_{\mathbb{A}}\right] \otimes_{\mathbb{A}} \mathbb{C}$ is an indecomposable and usually reducible module for the enveloping algebra $\mathbb{U}(\widehat{\mathfrak{g}})$ and hence also for the current algebra $\mathfrak{g}[t]$. Moreover if we pull-back this representation via the automorphism of $\mathfrak{g}[t] \rightarrow \mathfrak{g}[t]$ sending $x \otimes t^{r} \rightarrow x \otimes(t-1)^{r}, x \in \mathfrak{g}, r \in \mathbb{Z}_{+}$we get a representation $\left[\boldsymbol{\pi}_{\mathbb{C}}\right]$ of $\mathfrak{g}[t]$ and

$$
\left(\mathfrak{g} \otimes t^{N} \mathbb{C}[t]\right)\left[\boldsymbol{\pi}_{\mathbb{C}}\right]=0, \quad N>>0 .
$$

3.3. We now explain how to deduce the following result.

Theorem. For $\boldsymbol{\pi} \in \mathcal{P}_{\mathbb{Z}}^{+}(1)$, there exists an isomorphism of $\mathfrak{g}[t]$-modules

$$
\left[\boldsymbol{\pi}_{\mathbb{C}}\right] \cong D\left(\lambda_{1}, \lambda_{2}\right)
$$

where wt $\boldsymbol{\pi}=\lambda$ and $\left(\lambda_{1}, \lambda_{2}\right)$ is the interlacing pair associated to $\lambda$. In particular $\left[\boldsymbol{\pi}_{\mathbb{C}}\right]$ acquires the structure of a graded $\mathfrak{g}[t]$-module. 
The theorem can be proved by the same methods as the ones used in [4] for $\mathfrak{s l}_{n+1}$. It uses the following idea developed in [27] where similar questions were studied for a different family of irreducible representations.

Suppose that $\boldsymbol{\pi}_{1}, \boldsymbol{\pi}_{2} \in \mathcal{P}_{\mathbb{Z}}^{+}$are such that we have an injective map of $\mathbb{U}_{q}(\widehat{\mathfrak{g}})$-modules $\left[\boldsymbol{\pi}_{1} \boldsymbol{\pi}_{2}\right] \rightarrow\left[\boldsymbol{\pi}_{1}\right] \otimes\left[\boldsymbol{\pi}_{2}\right]$. Since $\mathbb{U}_{\mathbb{A}}(\widehat{\mathfrak{g}})$ is a Hopf subalgebra of $\mathbb{U}_{q}(\widehat{\mathfrak{g}})$, we get an injective map $\left[\left(\boldsymbol{\pi}_{1} \boldsymbol{\pi}_{2}\right)_{\mathbb{A}}\right] \rightarrow\left[\left(\boldsymbol{\pi}_{1}\right)_{\mathbb{A}}\right] \otimes\left[\left(\boldsymbol{\pi}_{2}\right)_{\mathbb{A}}\right]$. It was shown in [27, Lemma 2.20, Proposition 3.21] that tensoring with $\otimes_{\mathbb{A}} \mathbb{C}$ and pulling back by the automorphism of $\mathfrak{g}[t]$ induced by $t \rightarrow t-1$, gives rise to a map of $\mathfrak{g}[t]$-modules $\left[\boldsymbol{\pi}_{\mathbb{C}}\right] \rightarrow\left[\left(\boldsymbol{\pi}_{1}\right)_{\mathbb{C}}\right] \otimes\left[\left(\boldsymbol{\pi}_{2}\right)_{\mathbb{C}}\right]$. Here are the main steps in proving the theorem. Retain the notation of the theorem.

We prove that the choice of parameters in Definition 3.1 along with Theorem 1.9 implies that there exists a surjective map of $\mathfrak{g}[t]$-modules $D\left(\lambda_{1}, \lambda_{2}\right) \rightarrow\left[\boldsymbol{\pi}_{\mathbb{C}}\right] \rightarrow 0$.

Next note that one can define canonically, elements $\boldsymbol{\pi}_{1}, \boldsymbol{\pi}_{2} \in \mathcal{P}_{\mathbb{Z}}^{+}(1)$ with wt $\boldsymbol{\pi}_{s}=\lambda_{s}, s=1,2$ and $\boldsymbol{\pi}=\boldsymbol{\pi}_{1} \boldsymbol{\pi}_{2}$. Moreover, using the results in $([9,15])$, one has the following isomorphisms of graded $\mathfrak{g}[t]$-modules,

$$
\left[\left(\boldsymbol{\pi}_{1}\right)_{\mathbb{C}}\right] \cong_{\mathfrak{g}[t]} D\left(1, \lambda_{1}\right), \quad\left[\left(\boldsymbol{\pi}_{2}\right)_{\mathbb{C}}\right] \cong_{\mathfrak{g}[t]} D\left(1, \lambda_{2}\right) .
$$

The results in [6] show that there is an injective map of $\mathbb{U}_{q}(\widehat{\mathfrak{g}})$-modules $[\boldsymbol{\pi}] \rightarrow\left[\boldsymbol{\pi}_{1}\right] \otimes\left[\boldsymbol{\pi}_{2}\right]$. As discussed earlier, one can use [27, Lemma 2.20, Proposition 3.21] to show that the image of the induced map

$$
\left[\boldsymbol{\pi}_{\mathbb{C}}\right] \rightarrow\left[\left(\boldsymbol{\pi}_{1}\right)_{\mathbb{C}}\right] \otimes\left[\left(\boldsymbol{\pi}_{2}\right)_{\mathbb{C}}\right] \cong D\left(1, \lambda_{1}\right) \otimes D\left(1, \lambda_{2}\right)
$$

is $D\left(\lambda_{1}, \lambda_{2}\right)$. Hence we have a composition of surjective maps

$$
D\left(\lambda_{1}, \lambda_{2}\right) \rightarrow\left[\boldsymbol{\pi}_{\mathbb{C}}\right] \rightarrow D\left(\lambda_{1}, \lambda_{2}\right) .
$$

and Theorem 3.3 is proved.

\subsection{The connection with the category $\mathcal{C}_{\xi}$.}

We discuss the relationship of our work with that of [19, 20, 31] and restrict ourselves to $D_{n}$. Let $\xi:\{1,2, \cdots, n\} \rightarrow \mathbb{Z}$ and define the bipartite quiver on the Dynkin diagram of $D_{n}$ given by

$$
\xi(i)=\xi(i+1) \pm 1, \quad \xi(i)=\xi(i+2), \quad \xi(n-1)=\xi(n) .
$$

Let $\mathcal{C}_{\xi}$ be the full subcategory of finite-dimensional representations of $\mathbb{U}_{q}(\widehat{\mathfrak{g}})$ defined as follows: an object of $\mathcal{C}_{\xi}$ has all its Jordan-Holder components of the form $[\boldsymbol{\pi}]$, where $\boldsymbol{\pi} \in \mathcal{P}^{+}$is a product of terms of the form $\boldsymbol{\omega}_{i, a}, a \in\left\{q^{\xi(i) \pm 1}\right\}, 1 \leq i \leq n$. The following was proved in [19, 20] for $D_{4}$ and in [31] for $D_{n}$.

Theorem. The category $\mathcal{C}_{\xi}$ is closed under taking tensor products. The Grothendieck ring of $\mathcal{C}_{\xi}$ is a monoidal categorification of a cluster algebra of type $D_{n}$ with $n$ frozen variables.

Recall that the cluster variables in a cluster algebra of type $D_{n}$ are indexed by elements of $R^{+} \cup\left\{-\alpha_{i}: 1 \leq i \leq n\right\}$. The cluster variable associated to a root $\alpha_{i, j}$ of $D_{n}$ corresponds to a representation $[\boldsymbol{\pi}]$ where $\boldsymbol{\pi} \in \mathcal{P}_{\mathbb{Z}}^{+}(1)$ is given by $\boldsymbol{\pi}=\boldsymbol{\omega}_{i, a_{i}} \boldsymbol{\omega}_{i+1, a_{i+1}} \cdots \boldsymbol{\omega}_{j, a_{j}}$ and the $a_{k} s$ are determined by requiring that $\boldsymbol{\pi} \in \mathcal{P}_{\mathbb{Z}}^{+}(1)$ and $a_{i}=q^{\xi(i) \pm 1}$ if $\xi(i)=\xi(i-1) \pm 1$. Theorem 3.3 
gives a character formula for the prime objects corresponding to these cluster variables. More generally if we relax the condition that $\xi$ define a bipartite quiver, then the elements of $\mathcal{P}_{\mathbb{Z}}^{+}(1)$ should correspond to cluster variables in a suitable monoidal categorification. This was done in detail for $A_{n}$ in [3] and it was non trivial to identify the cluster variable corresponding to $[\boldsymbol{\pi}]$ when $\xi$ is not bipartite or monotonic.

For $D_{n}$, even in the bipartite case, it seems to be much more difficult to give the character of the prime representations corresponding to roots of the form $\beta_{i, j}$. However, preliminary calculations suggest that the graded limits of these will also admit a flag where the successive quotients are Demazure modules, but possibly of level bigger than two. We hope to return to these problems elsewhere.

\section{REFERENCES}

[1] Rekha Biswal, Vyjayanthi Chari, Peri Shereen and Jeffrey Wand, Macdonald Polynomials and level two Demazure modules for affine $\mathfrak{s l}_{n+1},(2019)$ arXiv:1910.05848.

[2] M, Brito and F. Pereira Graded Limits of Simple Tensor Product of KirillovReshetikhin Modules for $U_{q}\left(\tilde{\mathfrak{s l}}_{n+1}\right)$, Comm. in Algebra, 44, 10, (2016), 4504-4518.

[3] M. Brito, and V. Chari, Tensor products and q-characters of HL-modules and monoidal categorifications, Journal de l'cole Polytechnique Mathmatiques, 6, (2019), 581-619.

[4] M. Brito, V. Chari, and A. Moura,Demazure modules of level two and prime representations of quantum affine $s l_{n+1}$, J. Inst. Math. Jussieu, 31 pages, 2015.

[5] V. Chari, On the fermionic formula and the Kirillov-Reshetikhin conjecture, Int. Math. Res. Notices 12 (2001), 629-654.

[6] V. Chari, Braid group actions and tensor products, Int. Math. Res. Notices (2002), 357-382.

[7] V. Chari and S. Loktev, Weyl, Demazure and fusion modules for the current algebra of sl $l_{r+1}$, Adv. Math. 207 (2006), no.2, 928-960

[8] V. Chari and A. Moura, The restricted Kirillov-Reshetikhin modules for the current and twisted current algebras, Comm. Math. Phys. 266 (2006), no. 2, 431-454.

[9] V. Chari, A. Pressley, Weyl Modules for Classical and Quantum Affine Algebras Reprsent Theory, 5 (2001), 191-223.

[10] V. Chari and R. Venkatesh, Demazure modules, fusion products and Q-systems,Comm. Math. Phy. 333 (2), (2015), 799-830, no. 1, 191216.

[11] P. Di Francesco and R. Kedem, Proof of the combinatorial Kirillov-Reshetikhin conjecture, Int. Math. Res. Not. IMRN 7 (2008).

[12] E. Frenkel and E. Mukhin, Combinatorics of q-Characters of Finite-Dimensional Representations of Quantum Affine Algebras, Comm. Math. Phy. 216, no. 1, (2001), pp 23-57.

[13] E. Frenkel and E. Mukhin, The Hopf algebra RepU $U_{q}\left(g l_{\infty}\right)$, Selecta Math. (N.S.) 8, no. 4, (2002), 537635.

[14] E. Frenkel and N. Reshetikhin, The q-Characters of Representations of Quantum Affine Algebras and Deformations of $W$-Algebras, Recent Developments in Quantum Affine Algebras and related topics, Cont. Math., vol. 248, (1999), 163205.

[15] G. Fourier, P. Littelmann, Weyl modules, Demazure modules, KR-modules, crystals, fusion products and limit constructions, Adv. Math., 211 (2)(2007), pp. 566-593.

[16] G. Hatayama, A. Kuniba, M. Okado, T. Takagi and Z. Tsuboi, Paths, crystals and fermionic formulae, MathPhys odyssey, 2001, Prog. Math. Phys., 23, Birkhauser Boston, Boston, MA, (2002), 205272,

[17] G. Hatayama, A. Kuniba, M. Okado, T. Takagi and Y. Yamada, Remarks on fermionic formula, in Recent developments in quantum affine algebras and related topics, (Raleigh, NC, 1998), Contemp. Math., 248, Amer. Math. Soc., Providence, RI (1999) 243291.

[18] D. Hernandez, The Kirillov-Reshetikhin conjecture and solutions of T-systems, J. Reine Angew. Math. 596, (2006), 6387/

[19] D. Hernandez and B. Leclerc, Cluster algebras and quantum affine algebras, Duke Math. J. 154 (2010), 265-341, DOI 10.1215/00127094-2010-040. 
[20] D. Hernandez and B. Leclerc, Monoidal categorifications of cluster algebras of type A and D, Symmetries, Integrable Systems and Representations, Springer Proceedings in Mathematics \& Statistics 40 (2013), 175193. Boston, Boston, MA, 2006, 131169.

[21] R. Kedem, A pentagon of identities, graded tensor products, and the Kirillov-Reshetikhin conjecture, New trends in quantum integrable systems, World Sci. Publ. (2011), 173-193.

[22] A.N. Kirillov and N. Reshetikhin, Representations of Yangians and multiplicities of the inclusion of the irreducible components of the tensor product of representations of simple Lie algebras, J. Soviet Math. 52, no. 3, 31563164 (1990); translated from Zap. Nauchn. Sem. Leningrad. Otdel. Mat. Inst. Steklov. (LOMI) 160, Anal. Teor. Chisel i Teor. Funktsii. 8, 211221, 301 (1987).

[23] S. Kumar, Proof of the Parthasarathy-Ranga Rao-Varadarajan conjecture, Invent. Math. 93 (1988), 117130.

[24] C. Lenart, S. Naito, D. Sagaki, A. Schilling, and M. Shimozono, A uniform model for Kirillov-Reshetikhin crystals I: Lifting the parabolic quantum Bruhat graph, Int. Math. Res. Not., no. 7, (201, 18481901.

[25] J.R. Li and K. Naoi, Graded limits of minimal affinizations over the quantum loop algebra of type $G_{2}$, Algebras and Representation Theory 194 (2016), 957-973.

[26] O. Mathieu, Construction dun groupe de Kac-Moody et applications, Compositio Math. 69 (1989), no. 1, 3760.

[27] A. Moura, Restricted limits of minimal affinizations, Pacific J. Math. 244 (2010), 359-397.

[28] A. Moura and F. Pereira, Graded limits of minimal affinizations and beyond: the multiplicity free case for type $E_{6}$, Algebra and Discrete Mathematics 12 (2011), 69-115.

[29] H. Nakajima, Quiver Varieties and t-Analogs of q-Characters of Quantum Affine Algebras, Ann. of Math. 160 (2004), 1057 - 1097.

[30] H. Nakajima, t-analogs of q-characters of Kirillov-Reshetikhin modules of quantum affine algebras, Represent. Theory 7, (2003) 259274.

[31] H. Nakajima,Quiver varieties and cluster algebras, Kyoto J. Math.51 (2011), 71-126.

[32] K. Naoi, Fusion products of Kirillov-Reshetikhin modules and the $X=M$ conjecture, Adv. Math. 231 (2012), 1546-1571.

[33] _ Demazure modules and graded limits of minimal affinizations, Represent. Theory 17 (2013), 524556.

[34] - Graded limits of minimal affinizations in type D, SIGMA 10 (2014), 047, 20 pages.

[35] Defining relations of fusion products and Schur positivity, Toyama Mathmatical Journal, 37 (2015), 87-106.

[36] B. Ravinder, Generalized Demazure modules and fusion products, J. Algebra, 476 (2017), 186215.

[37] M. Okado and A. Schilling, Existence of Kirillov-Reshetikhin crystals for nonexceptional types. Represent. Theory 12 (2008), 186207.

Department of Mathematics, University of California, Riverside, 900 University Ave., RiverSIDE, CA 92521

E-mail address: chari@math.ucr.edu

Department of Mathematics, University of California, Riverside, 900 University Ave., RiverSIDE, CA 92521

E-mail address: jdavis@math.ucr.edu

Department of Mathematics, Ithaca College, 953 Danby Rd., Ithaca, Ny, 14850

E-mail address: rmoruzzi@ithaca.edu 